\title{
Modelling the Milky Way through adiabatic compression of cold dark matter haloes
}

\author{
V. F. Cardone ${ }^{1}$ and M. Sereno ${ }^{2,3}$ \\ 1 Dipartimento di Fisica "E.R. Caianiello", Università di Salerno, and INFN, Sez. di Napoli, Gruppo Coll. di Salerno, \\ via S. Allende, 84081 Baronissi (Salerno), Italy \\ e-mail: winny@na.infn.it \\ 2 Dipartimento di Scienze Fisiche, Università di Napoli, and INFN, Sez. di Napoli, Complesso Universitario di Monte \\ S. Angelo, via Cinthia, 80126 Napoli, Italy \\ 3 Istituto Nazionale di Astrofisica - Osservatorio Astronomico di Capodimonte, Salita Moiariello 16, 80131 Napoli, Italy
}

Received 21 September 2004 / Accepted 19 January 2005

\begin{abstract}
We use the adiabatic compression theory to build a physically well-motivated Milky Way mass model in agreement with observational data. The visible mass of the Galaxy is distributed in a spheroidal bulge and a multi-component disc parametrized by three galactic parameters, the Sun distance to the galactic centre, $R_{0}$, the total bulge mass, $M_{\text {bulge, and the }}$ local disc surface density, $\Sigma_{\odot}$. To model the dark matter component, we adiabatically compress a Navarro, Frenk and White (NFW) halo (with concentration $c$ and total mass $M_{\text {vir }}$ ) for fixed values of the spin parameter $\lambda$, the fraction of the mass in baryons $m_{\mathrm{b}}$, and the thin disc contribution to total angular momentum $j_{\mathrm{d}}$. An iterative selection procedure is used to explore in detail the wide space of parameters only selecting those combinations of $\left\{R_{0}, M_{\text {bulge }}, \Sigma_{\odot}, \lambda, m_{\mathrm{b}}, j_{\mathrm{d}}, c, M_{\text {vir }}\right\}$ that give rise to a Milky Way model in agreement with observational constraints. This analysis leads us to conclude that only models with $R_{0}=8.5 \mathrm{kpc}, 0.8 \times 10^{10} M_{\odot}<M_{\text {bulge }}<1.6 \times 10^{10} M_{\odot}$ and $49 M_{\odot} \mathrm{pc}^{-2} \leq \Sigma_{\odot} \leq 56 M_{\odot} \mathrm{pc}^{-2}$ can be reconciled with the set of observational constraints. As regards the parameters entering the adiabatic compression, we find $0.03 \leq \lambda \leq 0.10$ and $0.04 \leq m_{\mathrm{b}} \leq 0.10$, while final estimates of the parameters describing the initial halo profile turn out to be $5 \lessgtr c \lesssim 12$ and $7 \times 10^{11} M_{\odot} \lessgtr M_{\text {vir }} \lesssim 17 \times 10^{11} M_{\odot}($ all at $95.7 \% \mathrm{CL})$.
\end{abstract}

Key words. Galaxy: kinematics and dynamics - Galaxy: structure - galaxies: formation - dark matter

\section{Introduction}

Determining the mass distribution of the Milky Way is a classical task of astronomy (Schmidt 1956; Caldwell \& Ostriker 1981; Dehnen \& Binney 1998), while the usual framework for the origin of structures in the Universe is provided by the Cold Dark Matter (CDM) paradigm. This standard cosmological theory has proved very successful on a large scale by explaining both the abundance and clustering of galaxies (Peacock et al. 2001; Verde et al. 2002) and the power spectrum of the cosmic microwave background anisotropies (de Bernardis et al. 2000), but is now experiencing a number of difficulties when used on the scale of galaxies and dwarf galaxies. CDM paradigm predicts an over-abundance of satellites around the Milky Way and M 31 by an order of magnitude (Klypin et al. 1999; Moore et al. 1999). While the presence of a photoionizing background can solve this "sub-structure" problem (Somerville 2002), difficulty with the density profile still remains. Dark matter dominated objects, such as dwarf and low surface brightness galaxies, show rotation curves inconsistent with the central density cusp predicted by CDM cosmology (McGaugh \& de Block 1998; Alam et al. 2002).
The explanation of galaxy formation also remains unsolved in the standard hierarchical model. Dissipationless CDM haloes are assumed to form bottom-up via gravitational amplification of initial density fluctuations. Gas carried with such haloes cools and contracts within them to form luminous, independent self-gravitating units that can form stars at the halo centres (Fall \& Efstathiou 1980; Blumenthal et al. 1986; Mo et al. 1998). The halo profile affects both gas cooling and infall since it determines the structural properties of the resultant heavy discs and dense nuclear bulges. Even if the growth of dark haloes is not affected much by the baryonic components, the halo gravitationally responds to the dissipative baryonic infall, so the present day CDM haloes can have very different density profiles from the original CDM prediction.

In this context, we want to address the density profile problem by fitting CDM models to the observed properties of the Milky Way. The Milky Way seems to be a typical system on a mass scale of $10^{12} M_{\odot}$, mostly from contributions of exotic particles, such as weakly interacting massive particles or axions. Direct searches for dark compact objects such as MACHOs in the Milky Way halo have been performed by the MACHO and EROS collaborations through microlensing surveys. According to the MACHO group (Alcock et al. 2000b), the most likely 
halo fraction in the form of compact objects with mass in the range $0.1-1 M_{\odot}$ is of about $20 \%$; the EROS collaboration (Lasserre et al. 2000) has set a 95\% confidence limit that objects less than $1 M_{\odot}$ contribute less than $40 \%$ to the dark halo. These upper limits on the fraction of compact objects are evidences favouring that the Galaxy halo can be described by the standard cosmological approach.

Very detailed data obtained from many independent techniques to characterize the Milky Way make it a unique test for theories of galactic structure and baryonic infall. Actually, this consideration motivated Klypin et al. (2002) to perform analysis similar to the one we present in this paper. Although conceptually analogous to that of Klypin et al. (2002), the procedure we will use is much more detailed and allows deep exploration of the whole parameter space. As a result, we will be able not only to investigate the viability of the standard CDM paradigm when applied to the Galaxy, but also to constrain a set of parameters that are instead held fixed in the work of Klypin et al. (2002).

The paper is organized as follows. In Sect. 2 we describe the ingredients needed for our analysis and in Sect. 3 we briefly introduce the adiabatic compression theory. Section 4 is devoted to the description of the observational constraints on the Milky Way, while in Sect. 5 we explain how we investigate the parameter space to select only models in agreement with the data. The impact of the different selection criteria on the parameter space is discussed in Sect. 6 and results of our analysis are reported in Sect. 7. Section 8 is devoted to some final considerations.

\section{Model ingredients}

Traditional models of spiral galaxies usually include at least three dynamical components: a spheroidal bulge, an exponential disc, and a dark halo. The lack of observational data precludes using more detailed multi-component mass models, since it is not possible to break degeneracy among the many parameters involved. However, due to Earth's privileged position, cinematic and photometric data on the Milky Way are numerous enough to require the use of a multi-component model in order to have a unified picture. To this aim, we model the mass distribution in the Milky Way introducing five components that we describe in detail in the following subsections: the bulge, the thin and thick discs, the interstellar medium disc, and the dark halo.

\subsection{The bulge}

The morphology of the Galactic bulge, defined as the spheroid within the galactic coordinates $|l|<20^{\circ}$ and $|b|<10^{\circ}$ region around the Galactic centre, is much harder to ascertain than that of the bulges in many external galaxies, because of obscuration by interstellar dust due to our position in the Galactic plane. In recent years, however, striking images of the Galactic bulge have been obtained in the near infrared (at wavelengths of 1.25, 2.2, 3.5, and $4.9 \mu \mathrm{m}$ ) by the DIRBE experiment on board the COBE satellite (Arendt et al. 1994; Weiland et al. 1994) allowing study of its structure with good accuracy. These images suggest that the stellar distribution in the bulge is bar-shaped, i.e. that the bulge is not rotationally symmetric. However, a barlike structure cannot be used since the standard adiabatic compression theory formally assumes that all the mass distributions have spherical symmetry. On the other hand, it is possible to achieve relatively good agreement with the near infrared photometric data by also using spheroidal models for the bulge (Kent et al. 1991; Dwek et al. 1995). Thus, we will describe the bulge as a spheroidal density distribution (Dehnen \& Binney 1998):

$\rho_{\mathrm{b}}=\rho_{0}\left(\frac{m}{r_{0}}\right)^{\gamma}\left(1+\frac{m}{r_{0}}\right)^{\gamma-\beta} \mathrm{e}^{-m^{2} / r_{\mathrm{t}}^{2}}$,

where

$m^{2} \equiv\left(R^{2}+z^{2} / q^{2}\right)^{1 / 2}$,

with $R$ the galactocentric radius and $z$ the height above the equatorial plane. Thus the density of the bulge is proportional to $r^{-\gamma}$ for $r \ll r_{0}$, and to $r^{-\beta}$ for $r_{0} \ll r \ll r_{\text {t }}$, and softly truncated at $r=r_{\mathrm{t}}$. Fitting the model to the observed infrared photometric $C O B E / D I R B E$ data yields values for four of the five bulge parameters (Dehnen \& Binney 1998):

$\beta=\gamma=1.8, q=0.6, r_{0}=1 \mathrm{kpc}, r_{\mathrm{t}}=1.9 \mathrm{kpc}$.

Density normalization $\rho_{0}$ is not determined from the fitting relation, but is easily related to the total mass of the bulge, $M_{\text {bulge }}$. By integrating Eq. (1), one gets

$M_{\mathrm{b}}(m)=0.518 M_{\text {bulge }} m^{1.2}{ }_{1} F_{1}\left[0.6,1.6,-0.27 m^{2}\right]$

where ${ }_{1} F_{1}$ is the hypergeometric function and $M_{\text {bulge }}$ is related to the central density $\rho_{0}\left(\right.$ in $\left.M_{\odot} / \mathrm{pc}^{3}\right)$ as:

$M_{\text {bulge }}=1.60851 \times 4 \pi q \rho_{0}$.

In the adiabatic compression formalism, all the galaxy components are assumed to be spherical. To this aim, describing the bulge mass distribution requires a modified "spherical" version of Eq. (3). A simple and reasonable way to solve this problem is to substitute Eq. (3) by:

$$
\begin{aligned}
M_{\mathrm{b}}^{\mathrm{sph}}(r) \simeq & M_{\text {bulge }} f(r), \\
f(r)= & 1-\mathrm{e}^{-1.867 r}\left(1+1.543 r+0.1898 r^{2}\right. \\
& \left.+0.6349 r^{3}-0.6109 r^{4}+0.1491 r^{5}-0.01126 r^{6}\right),
\end{aligned}
$$

where $r$ is now the usual spherical radius. The spherical mass distribution in Eq. (5) has been defined so that the mean density inside the spherical radius $r$ is the same as the one within the elliptical radius $m, M_{\mathrm{b}}(m)$, i.e.

$\frac{M_{\mathrm{b}}^{\mathrm{sph}}(r)}{4 / 3 \pi r^{3}}=\frac{M_{\mathrm{b}}(m)}{4 / 3 \pi q m^{3}}$.

Using Eq. (5) instead of Eq. (3) introduces a systematic error when describing the very inner regions of the Galaxy. Actually, this error is not expected to be serious since the bulge contribution to the total mass budget of the Galaxy is indeed small. Moreover, as we will see later, most of the observational constraints we use probe a region of the Galaxy that is so far from 
the inner bulge dominated region that its dynamical effect could be described even by modelling this component as a pointlike mass. However, to further reduce this effect, we will use Eq. (5) for the bulge mass distribution, but consider the exact rotation curve (Binney \& Tremaine 1987):

$v_{\mathrm{c}}^{2}(R)=4 \pi G q \int_{0}^{R} \frac{\rho_{\mathrm{b}}\left(m^{2}\right) m^{2} \mathrm{~d} m}{\sqrt{R^{2}-\left(1-q^{2}\right) m^{2}}}$.

To fully characterize the bulge we only need its total mass, $M_{\text {bulge. }}$. Dwek et al. (1995) found $M_{\text {bulge }}=(1.3 \pm$ $0.5) \times 10^{10} M_{\odot}$.

\subsection{The disc}

In contrast to the bulge, the structure of the Milky Way disc is quite easy to investigate given the large amount of available data. The disc is made up of three components, namely the thin and the thick stellar discs and the interstellar medium (ISM) disc. We model the two stellar discs with the usual double exponential profile (Freeman 1970; Dehnen \& Binney 1998)

$\rho_{\mathrm{d}}(R, z)=\frac{\Sigma_{\mathrm{d}}}{2 z_{\mathrm{d}}} \exp \left(-\frac{R}{R_{\mathrm{d}}}-\frac{|z|}{z_{\mathrm{d}}}\right)$.

The total mass of each stellar disc is $M_{\mathrm{d}}=2 \pi \Sigma_{\mathrm{d}} R_{\mathrm{d}}^{2}$. For the adiabatic compression, we will adopt a 3-D mass distribution such that

$M_{\mathrm{d}}(r)=2 \pi \int_{-\infty}^{+\infty} \int_{0}^{r} \rho_{\mathrm{d}}(R, z) R \mathrm{~d} R \mathrm{~d} z$.

In Eq. (9), $M_{\mathrm{d}}(r)$ has a spherical symmetry that is not formally correct since the disc is a highly flattened structure. Actually, this approximation introduces a negligible error as it is witnessed by the good agreement between the predictions of the adiabatic compression theory and the numerical simulations (Jesseit et al. 2002). However, to evaluate the disc rotation curve and the vertical force we use the original flattened density distribution given in Eq. (8) ${ }^{1}$.

Although the simple formula in Eq. (8) may fit the largescale structure of the stellar discs well, it is not able to reproduce the smaller scale density fluctuations, which are prevalent in the ISM component. Dame et al. (1987) have shown that there is very little interstellar matter between the nuclear disc at $200 \mathrm{pc}$ and the molecular ring at $R=4-5 \mathrm{kpc}$. These local fluctuations strongly affect estimation of the Oort constants. Hence, we do not assume an analytical expression for the ISM disc density profile, but we use a third order polynomial interpolation of the data in Table D1 of Olling \& Merrifield (2001) augmented by $23.8 \%$ to take account of the helium contribution by mass. The 3-D mass distribution of the ISM disc is then evaluated as in Eq. (9), while the ISM disc rotation curve was evaluated following the method described in Kochanek (2002).

To fully characterize the disc model, we have to fix both the geometrical parameters $\left(R_{\mathrm{d}}, z_{\mathrm{d}}\right)$ and a value for the central

${ }_{1}$ To this aim we use a $\mathrm{C}++$ code kindly provided us by W. Dehnen, which implements a modified multipole technique developed by Kuijken \& Dubinski (1994; see also Dehnen \& Binney 1998). (or the local) surface density. We fix the geometry of our stellar discs giving their scale-length and scale-height as follows (Dehnen \& Binney 1998):

thin disc: $R_{\mathrm{d}}=\kappa R_{0} \mathrm{kpc}, z_{\mathrm{d}}=180 \mathrm{pc}$;

thick disc: $R_{\mathrm{d}}=\kappa R_{0} \mathrm{kpc}, z_{\mathrm{d}}=1000 \mathrm{pc}$,

where $R_{0}$ is the distance of the Sun to the galactic centre and $\kappa=(0.30 \pm 0.05)$ a scaling constant (Dehnen \& Binney 1998). The exact value of $R_{0}$ is quite uncertain with most of the estimates ranging from 7.0 to $8.5 \mathrm{kpc}$ (Kerr \& Lynden-Bell 1986; Reid 1993; Olling \& Merrifield 2000, 2001). Given the importance of this parameter in modeling the Galaxy, we explore models with different values of $R_{0}$.

We use the following estimate for the local surface density (Kuijken \& Gilmore 1989):

$\Sigma_{\odot}=(48 \pm 8) M_{\odot} \mathrm{pc}^{-2}$.

$\Sigma_{\odot}$ accounts for the contribution of the discs (both stellar and ISM), not taking the halo contribution into account. The total mass of each sub-disc is fixed by specifying the fractional contribution of each one to $\Sigma_{\odot}$. Using the relations given in Dehnen \& Binney (1998), we fix:

$\Sigma_{\text {thin }}\left(R_{0}\right)=14 \Sigma_{\text {thick }}\left(R_{0}\right)$

while, for the ISM disc, the adopted value is (Olling \& Merrifield 2001):

$\Sigma_{\mathrm{ISM}}\left(R_{0}\right)=14.5 M_{\odot} \mathrm{pc}^{-2}$.

It is noteworthy that there are also other estimates of $\Sigma_{\odot}$ significantly lower than the one used here (Olling \& Merrifield 2001; Gerhard 2002).

\subsection{The halo}

Observational data may be fitted by a wide range of models, even unphysical ones which explains why there are a lot of different dark halo models that claims to describe the density profile of this component well. To obtain physically interesting models, it is thus important to impose constraints based on a physical theory of halo formation and to select models that are both compatible with the data and also physically well motivated. From this point of view, numerical simulations of galaxy formation in hierarchical CDM scenarios are very helpful since they predict the initial shape of the dark matter distribution. In this paper, we assume a NFW profile (Navarro et al. 1997) as initial dark matter halo. The main properties of the NFW model are:

$\rho(r) \equiv \frac{\rho_{\mathrm{s}}}{x(1+x)^{2}}, x=r / r_{\mathrm{s}}$

$M(r)=4 \pi \rho_{\mathrm{s}} r_{\mathrm{s}}^{3} f(x)=M_{\mathrm{vir}} f(x) / f(c)$,

$f(x) \equiv \ln (1+x)-\frac{x}{1+x}$

$c \equiv r_{\mathrm{vir}} / r_{\mathrm{s}}$, 
$M_{\mathrm{vir}}=\frac{4 \pi \delta_{\mathrm{th}}}{3} \Omega_{\mathrm{M}} \rho_{\text {crit }} r_{\mathrm{vir}}^{3}$,

where $c$ is the concentration parameter, $M_{\mathrm{vir}}$ the virial mass and $r_{\text {vir }}$ the virial radius ${ }^{2}$. The model is fully described by two independent parameters that we assume to be $c$ and $M_{\mathrm{vir}}$. A correlation between $c$ and $M_{\text {vir }}$ has been found in numerical simulations (Navarro et al. 1997; Bullock et al. 2001; Colin et al. 2004), but we do not use such a relation since it is affected by quite large scatter $(\sim 25 \%)$.

The NFW model is not the only model proposed to fit the results of numerical simulations. Some authors (Moore et al. 1998; Ghigna et al. 2000) have proposed models with a central slope steeper than the NFW one. However, the difference between these models and the NFW one is very small for radii larger than $0.5 \%-1 \%$ of the virial radius, and it is further washed out by the baryonic infall. For these reasons, we will not consider models different from the NFW one.

\section{Adiabatic compression}

The present dark matter halo has a different shape compared to the original NFW model since gravitational collapse of the baryonic matter, which forms both the bulge and the disc, changes the overall gravitational potential of the system. The halo structure is thus modified by forces of the collapsed baryons with a role played also by the angular momentum of the galactic components (baryons and CDM). The effects of the baryonic infall are treated here following the approach of the adiabatic compression (Blumenthal et al. 1986; Flores et al. 1993; Mo et al. 1998). If the disc is assembled slowly, we can assume that the halo responds adiabatically to any modification in the gravitational potential and that it remains spherical while contracting. The angular momentum of the dark matter particles is then conserved and a particle which is initially at a mean radius $r_{\mathrm{i}}$ ends up at a mean radius $r$ where:

$M_{\mathrm{f}}(r) r=M\left(r_{\mathrm{i}}\right) r_{\mathrm{i}}$

with $M\left(r_{\mathrm{i}}\right)$ the initial total mass distribution within $r_{\mathrm{i}}$ and $M_{\mathrm{f}}(r)$ the total final mass within $r . M_{\mathrm{f}}(r)$ is the sum of the dark matter inside the initial radius $r_{\mathrm{i}}$ and the mass contributed by the baryonic components. We thus have:

$M_{\mathrm{f}}(r)=\left(1-m_{\mathrm{b}}\right) M\left(r_{i}\right)+M_{\mathrm{bar}}(r)$

where $M_{\mathrm{bar}}(r)$ is the sum of contributions from the baryonic components and $m_{\mathrm{b}}$ the fraction of total mass that ends up in the visible components. Note that we are implicitly assuming that the baryons initially had the same mass distribution as the CDM particles and that those which do not form the luminous units still remain distributed as the CDM.

For a given rotation curve $v_{\mathrm{c}}(R)$, the angular momentum of the thin disc is

$J_{\mathrm{d}}=2 \pi \int_{0}^{r_{\mathrm{vir}}} v_{\mathrm{c}}(R) \Sigma_{\mathrm{d}}^{\mathrm{thin}}(R) R^{2} \mathrm{~d} R$.

${ }^{2}$ The virial radius is defined such that the mean density within $r_{\mathrm{vir}}$ is $\delta_{\text {th }}$ times the mean matter density of the universe $\bar{\rho}=\Omega_{\mathrm{M}} \rho_{\text {crit }}$. We assume a flat universe with $\left(\Omega_{\mathrm{M}}, \Omega_{\Lambda}, h\right)=(0.3,0.7,0.72)$ and $\delta_{\text {th }}=$ 337 (Bryan \& Norman 1998).
The upper integration limit can be set to infinity, since the disc surface density $\Sigma_{\mathrm{d}}(R)$ drops exponentially and $r_{\mathrm{vir}}$ is much larger than the disc scale-length. We assume $J_{\mathrm{d}}$ to be a fraction $j_{\mathrm{d}}$ of the initial angular momentum of the halo, $J$ (i.e. $J_{\mathrm{d}}=$ $\left.j_{\mathrm{d}} J\right) . J$ can be expressed in terms of a spin parameter $\lambda$ defined as

$\lambda \equiv J|E|^{1 / 2} G^{-1} M_{\mathrm{vir}}^{-5 / 2}$,

with $E$, the total energy of the NFW halo,

$E=-\frac{G M_{\mathrm{vir}}^{2}}{2 r_{\mathrm{vir}}} f_{\mathrm{c}}$.

Some simple algebra allows us to rewrite Eq. (17) as (Mo et al. 1998)

$R_{\mathrm{d}}=\frac{1}{\sqrt{2}}\left(\frac{j_{\mathrm{d}}}{m_{\mathrm{d}}}\right) \lambda r_{\mathrm{vir}} f_{\mathrm{c}}^{-1 / 2} f_{\mathrm{R}}\left(\lambda, c, m_{\mathrm{d}}, j_{\mathrm{d}}\right)$

with:

$f_{\mathrm{c}}=\frac{c}{2} \frac{1-1 /(1+c)^{2}-2(1+c)^{-1} \ln (1+c)}{[c /(1+c)-\ln (1+c)]^{2}}$,

$f_{\mathrm{R}}\left(\lambda, c, m_{\mathrm{d}}, j_{\mathrm{d}}\right)=2\left[\int_{0}^{\infty} \mathrm{e}^{-u} u^{2} \frac{v_{\mathrm{c}}\left(R_{\mathrm{d}} u\right)}{v_{\text {vir }}} \mathrm{d} u\right]^{-1}$.

In Eqs. (20) and (22), $m_{\mathrm{d}}$ is the fraction of the total mass competing to the thin disc, $u \equiv R / R_{\mathrm{d}}$, and $v_{\text {vir }}$ is the total circular velocity at virial radius $r_{\text {vir }}$. It is important to stress that the rotation curve entering Eq. (17) is the total one, i.e.

$v_{\mathrm{c}}^{2}(r)=v_{\mathrm{c}, \mathrm{bar}}^{2}(r)+v_{\mathrm{c}, \mathrm{DM}}^{2}(r)$,

where the first term is simply evaluated given the distribution of the baryonic components, while the latter is:

$v_{\mathrm{c}, \mathrm{DM}}^{2}(r)=G\left[M_{\mathrm{f}}(r)-M_{\mathrm{bar}}(r)\right] / r$.

The full set of equations allows one to determine the final distribution of DM particles provided that a model for the density profiles of the baryons has been assigned and the parameters $\left(\lambda, m_{\mathrm{b}}, m_{\mathrm{d}}, j_{\mathrm{d}}, c, M_{\mathrm{vir}}\right)$ have been fixed. We will see later in Sect. 4 that these parameters are not all independent since it is possible to find some physically motivated relations among some of them.

There is some debate about the validity of the adiabatic compression formalism. Jesseit et al. (2002) have found substantial agreement between the final dark matter distribution in numerically simulated haloes and the one predicted by the adiabatic compression approach. On the other hand, this result has been contradicted on the basis of a set of higher resolution numerical simulations recently carried out by Gnedin et al. (2004), according to whom the standard adiabatic compression formalism systematically overpredicts the dark matter density profile in the inner $5 \%$ of the virial radius. Only one of the eight simulations considered by Gnedin et al. however refers to a galactic rather than a cluster halo. The bottom panel of Fig. 4 in their paper shows that the adiabatic compression formalism overpredicts the dark matter density less than $\sim 10 \%$ at $r / r_{\text {vir }} \sim 0.1$, while the error quickly decreases for larger values of $r / r_{\text {vir. }}$. This error is much smaller than the uncertainties we have on observational quantities so that we are confident that using the standard adiabatic formalism does not introduce any bias into the results. 


\section{Observational constraints}

We seek to select mass models of the Milky Way that are physically well motivated and are in agreement with observational data. We discuss in this section the observational constraints used to test each model.

Only compact baryonic objects can cause microlensing events towards the Galactic bulge. The number of microlensing events observed towards the galactic bulge (Alcock et al. 2000a; Popowski et al. 2000) determines the minimum baryonic mass in the inner Galaxy that can yield the measured value of optical depth $\tau$. For an axisymmetric mass density that decreases moving vertically away from the plane, it is possible to demonstrate that the minimum baryonic mass is (Binney \& Evans 2001):

$M_{\mathrm{bar}}^{\min }=\frac{\mathrm{e} c^{2} z_{0} \tau}{G}$

where $z_{0} \simeq R_{0} \tan b$, with $b$ the galactic latitude of the field target. This is only a lower estimate of the minimum baryonic mass inside the solar circle, and it is quite independent of the density profile of the baryonic components. Using the latest measured value of the optical depth towards the Baade window from analysis of 52 events in which a clump giant is lensed, $\tau=$ $(2.0 \pm 0.4) \times 10^{-6}$ (Popowski et al. 2000), we can thus select only those sets of parameters $\left(R_{0}, M_{\text {bulge }}, \Sigma_{\odot}\right)$ which produce a baryonic mass within $R_{0}$ above what is predicted by Eq. (25). We implicitly assume that all observed microlensing events are due to stellar lenses, which is true only if there is no compact dark matter (such as MACHOs) in the disc. Should this assumption not be true, the minimum baryonic mass should be lowered. However, this constraint is not very selective, so that changing the value of $M_{\mathrm{bar}}^{\mathrm{min}}$ does not affect our results.

While microlensing towards the bulge provides constraints on the inner Galaxy, satellite dynamics and modeling of the Magellanic Clouds motion probe the Galaxy mass distribution on a large scale $(\simeq 50-100 \mathrm{kpc})$. Lin et al. (1995) have used the dynamics of the Magellanic Clouds to infer the mass of the Milky Way inside $100 \mathrm{kpc}$, and obtained $M(r<100 \mathrm{kpc})=$ $(5.5 \pm 1) \times 10^{11} M_{\odot}$. This is in good agreement with the value found by Kochanek (1996) using escape velocity and motions of the satellite galaxies which give $(5-8) \times 10^{11} M_{\odot}$. Taking the different techniques used into account along with a dependence of the results on halo modeling, we follow Dehnen \& Binney (1998) by assuming as our constraint:

$M(r<100 \mathrm{kpc})=(7.0 \pm 2.5) \times 10^{11} M_{\odot}$.

A third and quite efficient constraint is given by the Oort's constants defined as:

$A=\frac{1}{2}\left[\frac{v_{\mathrm{c}}(R)}{R}-\frac{\mathrm{d} v_{\mathrm{c}}(R)}{\mathrm{d} R}\right]$,

$B=-\frac{1}{2}\left[\frac{v_{\mathrm{c}}(R)}{R}+\frac{\mathrm{d} v_{\mathrm{c}}(R)}{\mathrm{d} R}\right]$.

Dehnen \& Binney (1998) have reviewed the estimates of the Oort's constants present in the literature finally proposing the following values:

$A=(14.5 \pm 1.5) \mathrm{km} \mathrm{s}^{-1} \mathrm{kpc}^{-1}$,

$$
\begin{aligned}
& B=(-12.5 \pm 2.0) \mathrm{km} \mathrm{s}^{-1} \mathrm{kpc}^{-1}, \\
& A-B=(27.0 \pm 1.5) \mathrm{km} \mathrm{s}^{-1} \mathrm{kpc}^{-1}
\end{aligned}
$$

From the definitions of the Oort's constants, it immediately turns out that $(A-B) R_{0}=v_{\mathrm{c}}\left(R_{0}\right)$. We can thus replace the constraints on $A-B$ with a constraint on the local circular velocity

$v_{\mathrm{c}}\left(R_{0}\right)=R_{0} \times(27.0 \pm 1.5) \mathrm{km} \mathrm{s}^{-1}$.

The vertical force $K_{z}$ at some height above the plane places a condition on the local mass distribution. Using $\mathrm{K}$ stars as a tracer population, Kuijken \& Gilmore $(1989,1991)$ have deduced:

$K_{z, 1.1} \equiv\left|K_{z}\left(R_{0}, 1.1 \mathrm{kpc}\right)\right|=2 \pi G \times(71 \pm 6) M_{\odot} \mathrm{pc}^{-2}$.

Formally, this estimate depends on the galactic constants $R_{0}$ and $v_{\mathrm{c}}\left(R_{0}\right)$, but Olling \& Merrifield (2001) have shown that the result is quite robust against variations in these parameters. Thus, we can use the previous value to constrain our models.

Finally, another constraint comes from the rotation curve of our Galaxy. We can reconstruct this quantity from measurements of the velocity field. For an axisymmetric galaxy, the radial velocity relative to the local standard of rest $v_{\mathrm{r}}$ of a circular orbiting object at galactic coordinates $(l, b)$ and galactocentric radius $R$ is related to the circular speed by

$W(R) \equiv \frac{v_{\mathrm{r}}}{\sin l \cos b}=\frac{R_{0}}{R} v_{\mathrm{c}}(R)-v_{\mathrm{c}}\left(R_{0}\right)$

with the following relation between $R$ and the distance $d$ to the object:

$R^{2}=d^{2} \cos ^{2} b+R_{0}^{2}-2 d R_{0} \cos b \cos l$.

Several studies are available with measurements of both $d$ and $v_{\mathrm{r}}$ for objects which ought to be on a nearly circular orbits, so that the Milky Way rotation curve $v_{\mathrm{c}}(R)$ can be reconstructed. Here we use the data on HII regions and molecular clouds in Brand \& Blitz (1993) and the ones on a sample of classical Cepheids in the outer disc obtained by Pont et al. (1997). Following Dehnen \& Binney (1998), we reject objects with either $155^{\circ} \leq l \leq 205^{\circ}$ or $W<0$ or $d<1 \mathrm{kpc}$ when $v_{\mathrm{r}}$ is very likely dominated by non-circular motions.

\section{Exploring parameter space}

To apply the formalism of adiabatic compression in order to find the current halo mass profile, we need the initial halo shape and the present density profile of the baryonic components. As discussed above, according to observational data, the baryons have been distributed in the bulge and in the three sub-discs.

We still have, however, an indetermination for the baryonic components since the Sun distance to the galactic centre $R_{0}$, the total mass of the bulge, $M_{\text {bulge }}$, the local surface density, $\Sigma_{\odot}$, and the discs scale-lengths (fixed by the scaling constant $\kappa$ ) are known only with uncertainties. As a first step, we consider a grid of models with $R_{0}$ ranging from 7.0 to $8.5 \mathrm{kpc}$ in steps of $0.5 \mathrm{kpc}$, with $M_{\text {bulge }}$ ranging from 0.80 to $1.80 \times 10^{10} M_{\odot}$ with a step of $0.125 \times 10^{10} M_{\odot}$, with $\Sigma_{\odot}$ from 40 to $56 M_{\odot} \mathrm{pc}^{-2}$ in steps of $1.6 M_{\odot} \mathrm{pc}^{-2}$, and with $\kappa$ from 0.25 to 0.35 in steps 
of 0.05. Among these models we select only the ones that pass the test of the minimum baryonic mass within $R_{0}$. We stress that changing the value of $M_{\mathrm{bar}}^{\mathrm{min}}$ to consider the (quite unlikely) possibility that some microlensing events are not due to stellar lenses have only a minor effect on this criterium. The lower is the value of $M_{\mathrm{bar}}^{\mathrm{min}}$, the higher is the number of models passing this preliminary test, but all of the models added by lowering $M_{\mathrm{bar}}^{\mathrm{min}}$ will be excluded in the end by the selection procedure we describe later.

The parameters $\left(\lambda, m_{\mathrm{b}}, m_{\mathrm{d}}, j_{\mathrm{d}}, c, M_{\mathrm{vir}}\right)$ characterize the dark halo and are needed to solve the adiabatic compression equations. Furthermore, they give scaling relations between the baryonic components and total mass distribution. First, we note that $m_{\mathrm{d}}$ can be expressed as function of $m_{\mathrm{b}}$ as

$m_{\mathrm{d}}=\frac{M_{\text {thin }}}{M_{\text {bulge }}+M_{\text {thin }}+M_{\text {thick }}+M_{\mathrm{ISM}}} \times m_{\mathrm{b}} \equiv f_{\mathrm{d}} m_{\mathrm{b}}$,

where $M_{\text {thin }}, M_{\text {thick }}$, and $M_{\text {ISM }}$ are, respectively, the total mass of the thin, thick and ISM disc. It is also possible to express the virial mass $M_{\text {vir }}$ as a function of $M_{\text {thin }}$ and $m_{\mathrm{b}}$ as (Mo et al. 1998):

$M_{\mathrm{vir}}=\frac{M_{\mathrm{thin}}}{m_{\mathrm{d}}}=\frac{M_{\mathrm{thin}}}{f_{\mathrm{d}} m_{\mathrm{b}}}$.

Equation (36) simply states that the final mass of the system is the same as the initial one. Thus, we are now reduced to only four parameters, namely $\left(\lambda, m_{\mathrm{b}}, j_{\mathrm{d}}, c\right)$. We can further reduce the number of parameters observing that Eqs. (15), (16), and (20) are a system of three independent equations which can be iteratively solved to determine the initial radius $r_{\mathrm{i}}(r)$, the final mass $M_{\mathrm{f}}(r)$, and one of the four parameters for fixed values of the remaining three ones. As we will see later, while there are some hints about the distribution of the other parameters, little is known about the value of concentration $c$. We have thus decided to solve the set of equations with respect to $\left(r_{\mathrm{i}}, M_{\mathrm{f}}, c\right)$ having fixed parameters $\left(\lambda, m_{\mathrm{b}}, j_{\mathrm{d}}\right)$. The equations are highly non linear and must be solved iteratively ${ }^{3}$, so that to speed up the calculations, we have imposed a priori that $c$ should be in the range $(5,25)$ which is quite a conservative estimate for spiral galaxies similar to the Milky Way (Jimenez et al. 2003).

To explore the space of parameters in detail, we build, for each model with given values of $\left(R_{0}, M_{\text {bulge }}, \Sigma_{\odot}, \kappa\right)$, a set of models individuated by the values of $\left(\lambda, m_{\mathrm{b}}, j_{\mathrm{d}}\right)$. To define the grid, we fix a value for spin parameter $\lambda$. The distribution of $\lambda$ for haloes generated in numerical $N$-body simulations is approximated well by a log-normal distribution with parameters nearly independent of the cosmological parameters, halo mass, and redshift (Barnes \& Efstathiou 1987; Lemson \& Kauffmann 1999; Vitvitska et al. 2002). Using the parameters in Vitvitska et al. (2001), the distribution maximum is at $\lambda=0.035$, while there is a $90 \%$ probability that $\lambda$ is in the range $(0.02,0.10)$. We

\footnotetext{
${ }^{3}$ Mo et al. (1998) proposed an approximate relation which can be used to directly estimate $c$ from Eq. (20) thus avoiding the iterative procedure. However, their approximation has been obtained by neglecting the bulge and by assuming a single exponential disc instead of the three sub-discs we are using. We checked that their formula may lead to strongly underestimate the value of $c$.
}

thus let $\lambda$ change in this range in steps of 0.01 . Next, we have to fix a value for $m_{\mathrm{b}}$. This parameter is poorly constrained since we only know that it cannot be larger than the universal baryon fraction $\Omega_{\mathrm{b}} / \Omega_{\mathrm{M}}$, inferred by observations involving completely different physical processes (Turner 2002). The power spectrum of matter inhomogeneities from observations of largescale structure is sensitive to $\Omega_{\mathrm{b}} / \Omega_{\mathrm{M}}$; the Two Degree Field Galaxy Redshift Survey has reported a value of $0.15 \pm 0.07$ (Percival et al. 2001). Measurements of the angular power spectrum of the CMBR also provide a very significant estimate. The combined analysis in Jaffe et al. (2001) of several datasets gives $\Omega_{\mathrm{b}} / \Omega_{\mathrm{M}}=0.186_{-0.008}^{+0.010}$. We thus let $m_{\mathrm{b}}$ change from 0.01 to 0.20 in steps of 0.01 . Finally, we have to fix $j_{\mathrm{d}}$. This parameter is also constrained neither theoretically nor by numerical simulations. On one hand, it is reasonable to assume $j_{\mathrm{d}}=m_{\mathrm{d}}$, which indeed seems necessary to fit spiral galaxy rotation curves (Mo et al. 1998). On the other hand, numerical simulations have found $j_{\mathrm{d}} / m_{\mathrm{d}}$ significantly less than unity. We therefore vary this parameter from 0.005 to $m_{\mathrm{d}}$ in steps of 0.01 .

The grid we build in this way is quite detailed. For each given $\left(R_{0}, M_{\text {bulge }}, \Sigma_{\odot}, \kappa\right)$ the total number of sets $\left(\lambda, m_{\mathrm{b}}, j_{\mathrm{d}}\right)$ is $\sim 800-1200$ (depending on the value of $m_{\mathrm{b}}$ ), so that the parameter space is indeed checked intensively.

To select among this large number of models we use a multi-step procedure for each model parameterization. First, we iteratively solve the set of Eqs. (15), (16) and (20) so that we have the full halo mass distribution. We are thus able to estimate $M_{\mathrm{f}}(r=100 \mathrm{kpc})$, the total mass inside $r=100 \mathrm{kpc}$. A model passes to the next step only if this value is consistent with the estimate in Eq. (26). In the second step, we compare the local circular velocity $v_{\mathrm{c}}\left(R_{0}\right)$ to the constraint in Eq. (32), and reject that model if there is no agreement. Next, the Oort's constants are evaluated and the model retained if the values of $A$ and $B$ are in agreement with the values given by Eqs. (29) and (30). Then, we compute $K_{z, 1.1}$ and accept the model if the resulting value is in agreement with the estimate reported in Eq. (33). For the surviving models, we estimate the $\chi^{2}$ defined as

$\chi^{2}=\frac{1}{N} \sum \frac{v_{\mathrm{c}, \text { data }}^{2}\left(R_{i}\right)-v_{\mathrm{c}, \text { model }}^{2}\left(R_{i}\right)}{\sigma_{i}^{2}}$,

where $\sigma_{i}$ is the error on the $i$ th measurements and the sum runs over the $N$ data points. Note that we compute the $\chi^{2}$ as the last step of our selection procedure when all the model parameters are fixed so that we do not change their values in order to minimize $\chi^{2}$. We deem a model acceptable if $\chi^{2}<1.33$. For our data set of $N=115$ entries, this corresponds to a $99.7 \%$ confidence level.

Actually, as noted by Olling \& Merrifield (2001), selecting among different models on the basis of the $\chi^{2}$ value is not a statistically correct procedure because the errors on both $v_{\mathrm{c}}(R)$ and the Galactic constants are not normal, as they enter the estimate of $v_{\mathrm{c}}(R)$ through Eq. (34). This means that a high value of $\chi^{2}$ for a given model might be a consequence of an intrinsically wrong model or of the not Gaussian origin of the errors. Furthermore, the data of Pont et al. (1997) on the radial velocities of outer disc classical Cepheids are given without any uncertainties, so that for these data points $\sigma_{i}$ is only determined by propagation 
of the errors on the galactic constants, so it is underestimated. As a result, we decided to still retain the $\chi^{2}$-test as a selection criterium, but also consider the median statistics. As shown in Gott et al. (2001; see also Avelino et al. 2002; Chen \& Ratra 2003; Sereno 2003), median statistics provide a powerful alternative to $\chi^{2}$ likelihood methods. Fewer assumptions about the data are needed. A proper median statistics assume that (i) experimental results are statistically independent; and (ii) there are no systematic effects. Statistical errors are not required to be either known or gaussianly distributed. Since our analysis is based on not Gaussian errors, performing a test without using the errors themselves turns out to be a very conservative approach. Furthermore, median statistics is also less vulnerable to the presence of bad data and outliers.

To compute the likelihood of a particular set of parameters, we count how many data points are above or below each model prediction and compute the binomial likelihoods. Given a binomial distribution, if we perform $N$ measurements, the probability of obtaining $k$ of themabove the median is given by

$P(k)=\frac{2^{-N} N !}{k !(N-k) !}$.

We count how many of the 115 experimental points are above the expected velocity rotation curve, and retain a model if the number of overestimates is between 43 and 72 . Given the distribution in Eq. (38), the probability that the median of 115 sorted entries falls in this range is $99.73 \%$.

\section{Analysis of the selection criteria}

Before presenting the results of our analysis, it helps to investigate at what stage in our selection procedure certain types of models are excluded; i.e. we want to study the impact of each criterium on the parameter space. For a given set of galactic parameters $\left(R_{0}, M_{\text {bulge }}, \Sigma_{\odot}, \kappa\right)$, we first select all the models with $c$ in the range $(5,25)$ and then apply to this set of models the selection criteria introduced in the previous section separately. The main results of this analysis are presented in Figs. 1-5 and discussed below as regards the two parameters entering the adiabatic compression equations, i.e. $c$ and $m_{\mathrm{b}}$.

- Constraint on $M(r<100 \mathrm{kpc})$. As Fig. 1 shows, application of this criterium tends to flatten the histogram of the $c$ values, but is slightly more effective in cutting out models with values of $c$ in the tails of the distribution. On the contrary, this cut tends to suppress the high end of the $m_{\mathrm{b}}$ histogram, and only retains those models with smaller $m_{\mathrm{b}}$. This latter result may be qualitatively explained by considering Eq. (36) which shows that the higher $m_{\mathrm{b}}$, the lower $M_{\mathrm{vir}}$ and thus $M(r<100 \mathrm{kpc})$.

- Constraint on $v_{\mathrm{c}}\left(R_{0}\right)$. Figure 2 shows the impact of the test on the local circular velocity. High values of $c$ are excluded by this constraint. As regards the $m_{\mathrm{b}}$ histogram, this selection criterium turns out to be almost orthogonal to the previous one since now low values of $m_{\mathrm{b}}$ are clearly disfavoured.

- Constraint on the Oort constants. This test turns out to be a sort of compromise between the two previous ones. On one hand, it is quite effective in excluding models with very
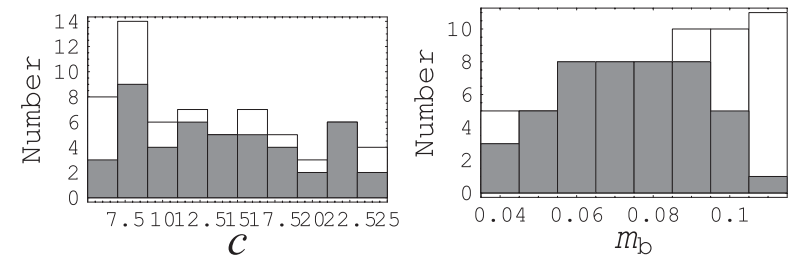

Fig. 1. Histogram of the number distribution of $c$ and of $m_{\mathrm{b}}$ for the models with $\left(R_{0}, M_{\text {bulge }}, \Sigma_{\odot}, \kappa\right)=(8.5,1.0,54,0.3)$ and $c$ between 5 and 25. $R_{0}$ is in kpc, $M_{\text {bulge }}$ in $10^{10} M_{\odot}, \Sigma_{\odot}$ in $M_{\odot} / \mathrm{pc}^{2}$. The shaded histogram refers to the models passing the test on $M(r<100 \mathrm{kpc})$.
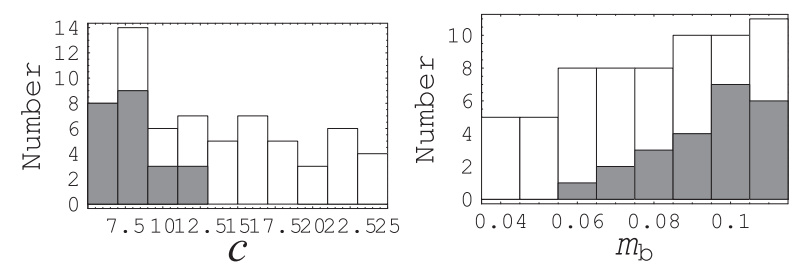

Fig. 2. Same as Fig. 1 for the test on $v_{\mathrm{c}}\left(R_{0}\right)$.
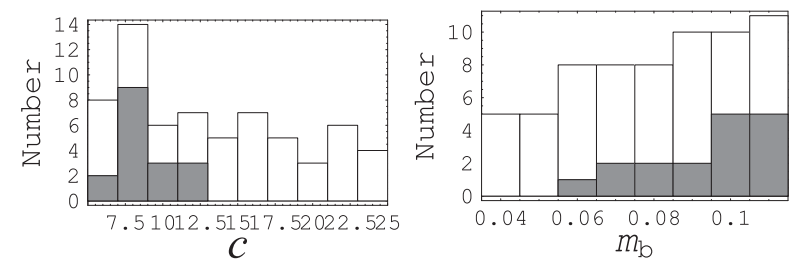

Fig. 3. Same as Fig. 1 for the test on the Oort constants.

low values of $c$ (look at the lowest bin), as it is (with much less efficiency) for the constraint on $M(r<100 \mathrm{kpc})$. On the other hand, it cuts away the low end of the $m_{\mathrm{b}}$ histogram in the same way as the selection criterium based on the value of $v_{\mathrm{c}}\left(R_{0}\right)$ do. This is quite an important result since it shows that the eventual exclusion of this constraint does not alter the final results of the multi-step selection procedure. The presence of local fluctuations in the ISM disc density strongly affects derivatives of the gravitational potential, hereby leading to possible errors in evaluation of the Oort constants for a given set of galactic parameters. One could thus argue that it would be better not to use the Oort constants as a selection criterium, but instead choose their difference and hence the local circular velocity. However, use of a third order polynomial interpolation of the measured ISM disc density alleviates this problem, so that we are confident that the estimated values of $A, B$ are not corrupted. Furthermore, as we have observed comparing Fig. 3 with Figs. 1 and 2, applying only the constraint on $A, B$ gives results that are consistent with those obtained using the two constraints on $M(r<100 \mathrm{kpc})$ and $v_{\mathrm{c}}\left(R_{0}\right)$, so that any systematic error in estimating the Oort constants is washed out in our multi-step procedure.

- Constraint on $K_{z, 1.1}$. This test works by excluding models with values of $c$ and $m_{\mathrm{b}}$ in the tails of the distribution. This constraint is very effective when selecting among different galactic parameters as it leads to rejecting models with $\Sigma_{\odot}<49 M_{\odot} / \mathrm{pc}^{2}$. Actually, the lower $\Sigma_{\odot}$, the higher the percentage of models excluded either by the test on 

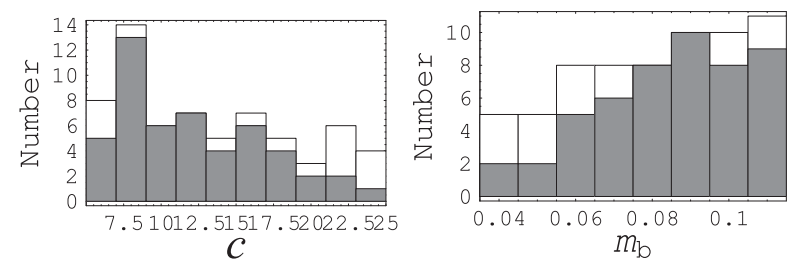

Fig. 4. Same as Fig. 1 for the test on $K_{z, 1.1}$.
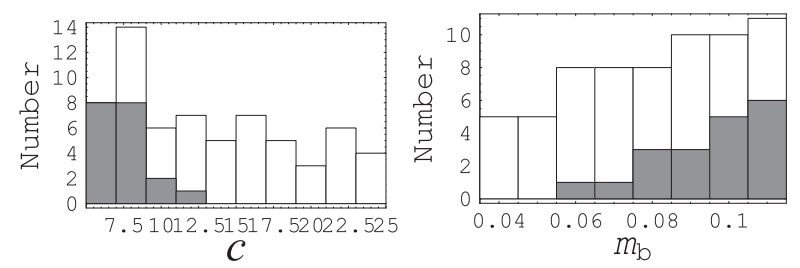

Fig. 5. Same as Fig. 1 for the test on $\chi^{2}$ value.

$M(r<100 \mathrm{kpc})$ or by that on the Oort constants. This is clear evidence against models with low values of the local surface density in agreement with claims about results applying of the constraint on $K_{z, 1.1}$.

- Constraint on $\chi^{2}$. Application of this constraint allows us to flattening the histogram of the $c$ values thus lowering the peak in Fig. 5 for low values. However, the high end of the histogram is erased hereby suggesting that models with very high values of $c$ are not able to fit the Milky Way rotation curve. This is not unexpected since both $N$-body simulations and fitting of the adiabatically compressed NFW model to external galaxies show that small values of $c$ are best suited to describe galactic dark haloes (Jimenez et al. 2003). As for $m_{\mathrm{b}}$, the constraint on $\chi^{2}$ works as the one on the local circular velocity by selecting models with high values for this parameter. This is a reasonable result since both constraints are related to the same physical quantity, i.e. the rotation curve. The median statistics works the same way as the $\chi^{2}$-test, but it is more stringent.

As a final remark, the observational data we have reviewed in Sect. 3 are "consensus" values with "consensus" errors that cannot therefore be treated as "statistical" uncertainties. This is the reason we decided to adopt a multi-step selection procedure instead of the usual $\chi^{2}$ minimization technique based on the definition of $\chi^{2}$ entering all the constraints at the same time. Our filtering approach and the discussion presented in this section allow one to avoid all the problems connected with the statistics of not Gaussian errors and makes it possible to understand how the results could vary by changing one of the constraints. Actually, the procedure we have implemented is quite robust since the different constraints select different regions of the parameter space, thus allowing the ranges to be narrowed for both the adiabatic compression parameters and the galactic constants.

\section{Results}

The selection procedure is quite efficient because it allows us to reject most of the models. Since we have used two alternative tests as a last constraint (the $\chi^{2}$ value or the median

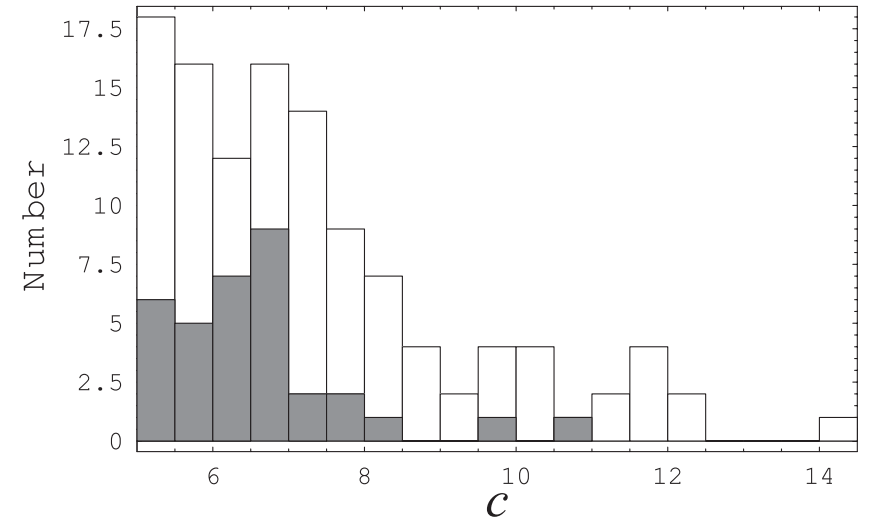

Fig. 6. Histogram of the number distribution of concentration parameter $c$ for models in Sample A and Sample B (shaded histogram).

Table 1. Median values, $68 \%$ and $95 \%$ regions for the model parameters of Sample A. The total halo mass $M_{\text {vir }}$ is expressed in units of $10^{11} M_{\odot}$.

\begin{tabular}{cccc}
\hline \hline Parameter & Median & 68\% range & 95\% range \\
\hline$c$ & 6.89 & $5.49-9.45$ & $5.04-11.88$ \\
$M_{\text {vir }}$ & 8.68 & $7.53-10.20$ & $6.87-16.60$ \\
$m_{\mathrm{b}}$ & 0.08 & $0.07-0.09$ & $0.04-0.10$ \\
$\lambda$ & 0.06 & $0.04-0.09$ & $0.03-0.10$ \\
$\lambda^{\prime}$ & 0.029 & $0.026-0.035$ & $0.018-0.042$ \\
\hline
\end{tabular}

statistics), we define two samples. Sample A contains those models passing all the selection criteria and having $\chi^{2}<1.33$, while Sample B is made up of the models passing the test only on the median statistics. The final number of models is 116 in Sample A and 34 in Sample B from an initial set of $\sim 10^{6}$. It turns out that Sample B is a subset of Sample A; i.e. all the models in Sample B belong to Sample A too. This is expected since the median statistics is a more restrictive test than $\chi^{2}$ analysis.

The main results are shown in Figs. 6-9 and summarized in Tables 1 and 2. The results obtained from the two samples are in perfect agreement, so we do not discuss them separately. We must, however, stress that the final samples of models may not be treated using the usual statistical methods, since our selection procedure is based on constraints on different observable quantities that are "consensus" values with "consensus" errors. That is why we do not report their mean values as a best estimate of the parameters, but instead report the medians which is a more conservative approach. Because of this, the quoted $68 \%$ $(95 \%)$ range must not be considered as the $1-\sigma(2-\sigma)$ confidence limit, but is simply the range that the $68 \%(95 \%)$ of the values are found within. With this caveat in mind, we discuss the distribution of the various model parameters below.

1. Figure 6 shows the distribution of the values of concentration parameter $c$. Small values are clearly favoured so that the median value for Sample A (Sample B) comes out as $c=6.89$ (6.44). The values of $c$ we get are lower than expected, but not unrealistic. Jimenez et al. (2003) have fitted the rotation curves of 400 spiral galaxies by modelling them 
Table 2. Median values, $68 \%$ and $95 \%$ regions for the model parameters of Sample B. The total halo mass $M_{\mathrm{vir}}$ is expressed in units of $10^{11} M_{\odot}$.

\begin{tabular}{cccc}
\hline \hline Parameter & Median & $68 \%$ range & $95 \%$ range \\
\hline$c$ & 6.48 & $5.49-7.26$ & $5.08-9.75$ \\
$M_{\text {vir }}$ & 8.44 & $7.58-9.39$ & $7.03-11.16$ \\
$m_{\mathrm{b}}$ & 0.08 & $0.07-0.09$ & $0.06-0.09$ \\
$\lambda$ & 0.06 & $0.04-0.09$ & $0.03-0.10$ \\
$\lambda^{\prime}$ & 0.029 & $0.027-0.032$ & $0.023-0.035$ \\
\hline
\end{tabular}

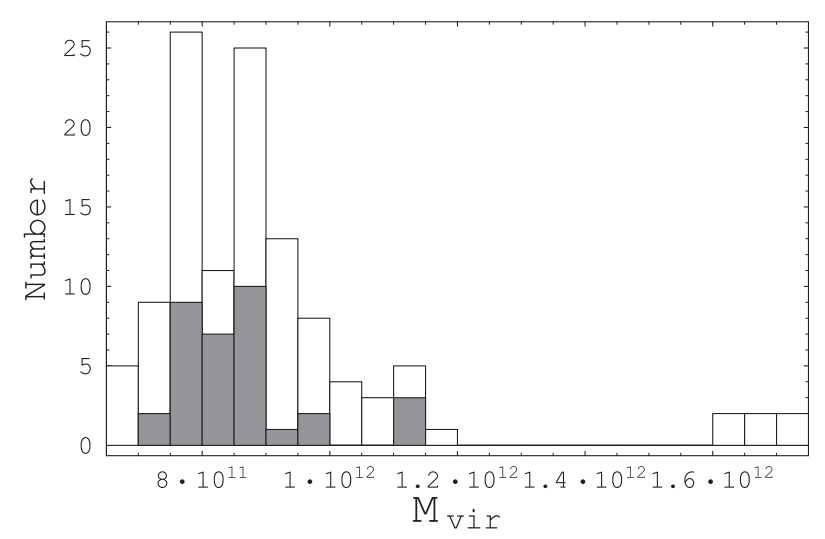

Fig. 7. Same as Fig. 6 for total halo mass $M_{\text {vir }}$.

with an exponential disc and a dark halo obtained by adiabatically compressing the NFW profile. Their Fig. 1 shows the distribution of the concentration $c$ vs. total mass $M_{\mathrm{vir}}$. There are indeed a lot of galaxies with values of $c$ in the same range as the one found here.

2. In Fig. 7, we plot the distribution of values of total mass $M_{\text {vir }}$ for the final set of models. The median value for Sample A (Sample B) is $8.68(8.44) \times 10^{11} M_{\odot}$, and the $95 \%$ confidence interval ranges from 5.04 (5.08) to $6.74(9.75) \times 10^{11} M_{\odot}$. These values are still in agreement with the most recent measurement of the Milky Way total mass estimated to be $1.9_{-1.7}^{+3.6} \times 10^{12} M_{\odot}$ (Wilkinson \& Evans 1999). It is noteworthy, however, that the value given by Evans \& Wilkinson is higher than usual considering that most of the previous estimates are lower than $10^{12} M_{\odot}$ (see, e.g., Fig. 3 in Zaritsky 1999). Sample A also contains very few models with very high values of $M_{\text {vir }}$. The $M_{\text {vir value }}$ we obtained may thus be considered quite reasonable. It is also possible to see that a clear correlation exists between $c$ and $M_{\mathrm{vir}}$, with the lower values of concentration parameter $c$ giving rise to the highest values of the total mass. This correlation may reflect the existence of some covariance among the model parameters in the analysis procedure.

3. Distribution of $m_{\mathrm{b}}$ is shown in Fig. 8. The median value is 0.08 for both Sample A and B, while the $95 \%$ range turns out to be $0.07-0.09$. According to some authors (Mo et al. 1998; Jimenez et al. 2003; Klypin et al. 2002), $m_{\mathrm{b}}$ should be written as $\varepsilon \times \Omega_{\mathrm{b}} / \Omega_{\mathrm{M}}$ with $\varepsilon$ indicating the efficiency of the transfer of baryons from the initial halo to the bulge and disc. If this were correct, our results would

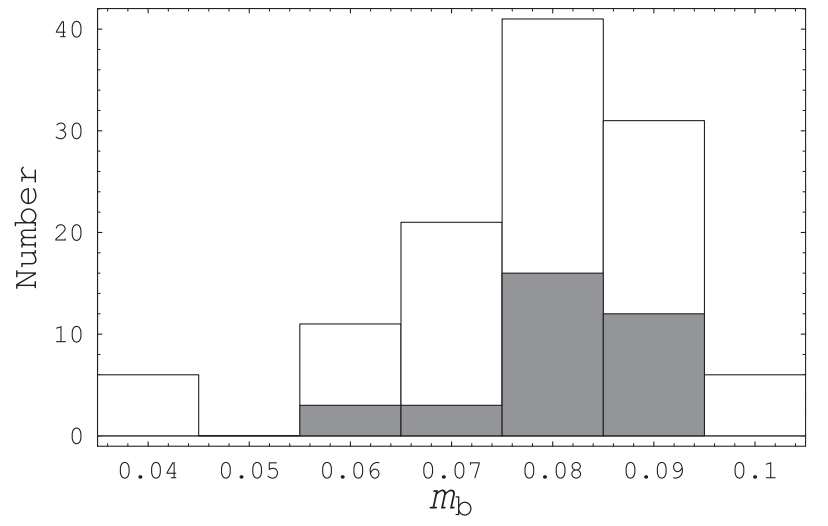

Fig. 8. Same as Fig. 6 for $m_{\mathrm{b}}$.
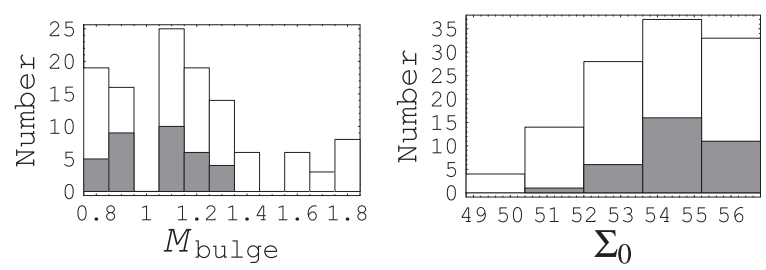

Fig. 9. Histograms of the number distribution of models according to values of $M_{\text {bulge }}\left(10^{10} M_{\odot}\right.$, left panel $)$ and $\Sigma_{\odot}\left(M_{\odot} \mathrm{pc}^{-2}\right.$, right panel $)$. Shaded regions refer to Sample B.

mean $\varepsilon \simeq 37-48 \%$ for $\Omega_{\mathrm{b}} / \Omega_{\mathrm{M}}=0.186$. Efficiency is not one which raises the problem of understanding where the missing baryons are.

4. The median value of spin parameter $\lambda$ is 0.06 for both Sample A and B, and the 95\% range is 0.04-0.09. Models with low spin are erased by the selection procedure. We do not report any result for $j_{\mathrm{d}}$ since this parameter is found to be degenerate with $\lambda$ and $m_{\mathrm{d}}$, i.e., for fixed values of $\left(m_{\mathrm{b}}, R_{0}, M_{\text {bulge }}, \Sigma_{\odot}, \kappa\right)$, models having the same value of $\lambda^{\prime}=\lambda j_{\mathrm{d}} / m_{\mathrm{d}}$ give rise to the same present day halo mass profile. This result is not unexpected since in the only equation containing $\lambda$ and $j_{\mathrm{d}}$, Eq. (20), these two parameters appear only in $\lambda^{\prime}$. The median value for this is $\lambda^{\prime}=0.029$ with a $95 \%$ range going from 0.018 to 0.042 for Sample A and from 0.023 to 0.035 for Sample B.

5. Figure 9 shows the histogram of the number of models according to the values of $M_{\text {bulge }}$ and $\Sigma_{\odot}$. We can draw some interesting limits. First, we observe that we are not able to significantly constraint the bulge total mass. Indeed, the median value of the distribution turns out to be $M_{\text {bulge }}=1.0 \times 10^{10} M_{\odot}$, but the $95 \%$ range for Sample A does not reject any model. Values of $M_{\text {bulge }}$ in the range $0.8-1.4 \times 10^{10} M_{\odot}$, however, seem to be favoured, as confirmed by analysing Sample B that excludes models with $M_{\text {bulge }}>1.4 \times 10^{10} M_{\odot}$. A similar analysis leads to the following constraints on the disc local surface density

$49 M_{\odot} \mathrm{pc}^{-2} \leq \Sigma_{\odot} \leq 56 M_{\odot} \mathrm{pc}^{-2}$

with $54 M_{\odot} \mathrm{pc}^{-2}$ as median value. If we subtract the contribution of the ISM disc surface density, we get for the stellar disc $\Sigma_{\star} \simeq 40 M_{\odot} \mathrm{pc}^{-2}$ in agreement with the consensus value $\left(\Sigma_{\star}=35 \pm 10 M_{\odot} \mathrm{pc}^{-2}\right)$ proposed by 


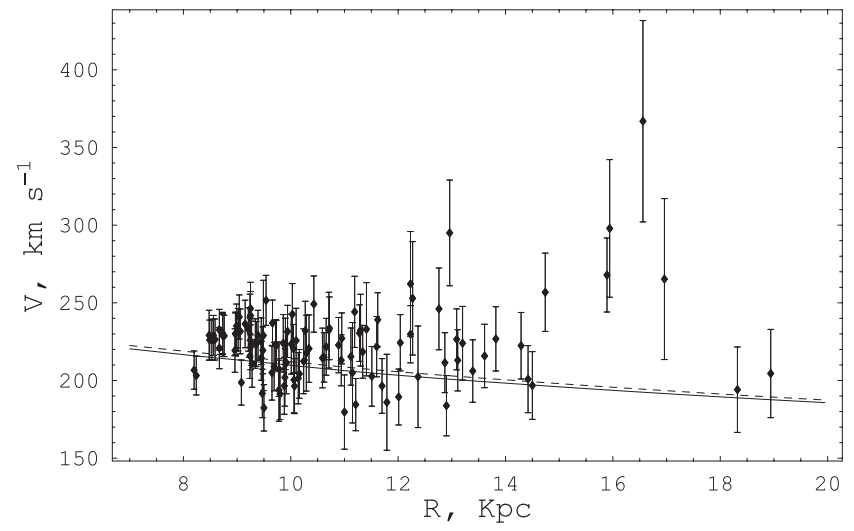

Fig. 10. Rotation velocity curve in the Galactic plane for the models $\left\{\Sigma_{\odot}, M_{\text {bulge }}, c, m_{\mathrm{b}}, j_{\mathrm{d}}, \lambda\right\}=\left\{54.4 M_{\odot} \mathrm{pc}^{-2}, 1.05 \times 10^{10} M_{\odot}, 5.55,0.08\right.$, $0.035,0.04\}$ (full line) and $\left\{\Sigma_{\odot}, M_{\text {bulge }}, c, m_{\mathrm{b}}, j_{\mathrm{d}}, \lambda\right\}=\left\{56.0 M_{\odot} \mathrm{pc}^{-2}\right.$, $\left.9.25 \times 10^{9} M_{\odot}, 6.56,0.09,0.045,0.04\right\}$ (dashed line).

Olling \& Merrifield (2001). Only 6 out of 116 models in Sample A have $R_{0}=8.0 \mathrm{kpc}$, while in all the other cases it is $R_{0}=8.5 \mathrm{kpc}$, so that we may safely consider $R_{0}=8.5 \mathrm{kpc}$ as our final estimate of this galactic constant. This conclusion is strengthened by noting that all the models in Sample B have $R_{0}=8.5 \mathrm{kpc}$. This value is somewhat higher than expected since most recent estimates predict values in the range 7.0-8.0 kpc (Reid 1993; Olling \& Merrifield 2000). However, the estimate of $R_{0}$ is somewhat model-dependent since its value is linked to halo flattening. For instance, Olling \& Merrifield (2001) have found that it is possible to build galaxy models with $R_{0} \gtrsim 7.0 \mathrm{kpc}$ if the halo is close to spherical, which indeed is our case. Finally, there are no models with $\kappa \neq 0.30$ so that we conclude that the discs scale-length is $R_{\mathrm{d}}=0.30 \times R_{0}=2.55 \mathrm{kpc}$. This is lower than the fiducial value $(3.5 \mathrm{kpc})$ suggested by Binney \& Tremaine (1987) and is often adopted in many Milky Way disc modelling. However, we note that a value of $R_{\mathrm{d}} \simeq 2.5$ is favoured by both star count models (Robin et al. 1992; Ortiz \& Lépine 1993) and the integrated NIR luminosity profile (Freudenreich 1998; Binney et al. 1997).

Finally, we again wish to comment on the use of $\chi^{2}$ as a selection criterium. Actually, it is difficult to say whether any value of $\chi^{2}$ for a given model is due to some systematic error, such as neglecting the triaxial structure of the bulge and the flattening of the halo, or to a problem with the parameter values or to the not Gaussian nature of the estimated errors on the rotation curve. However, agreement among the results obtained using both the $\chi^{2}$ analysis and the median statistics (that turns out to be a more selective criterium) is convincing evidence that the excluded models have been rejected as a consequence of physical problems, either due to the need for more careful description of the inner Galaxy or to an intrinsically wrong combinations of the parameters $\left(\lambda, m_{\mathrm{b}}, j_{\mathrm{d}}, c\right)$. This makes us confident the results. As an example, in Fig. 10 we plot the rotation curve in the Galactic plane for two models, one with the lowest value of $\chi^{2}\left(\left\{\Sigma_{\odot}, M_{\text {bulge }}, c, m_{\mathrm{b}}, j_{\mathrm{d}}, \lambda\right\}=\left\{54.4 M_{\odot} \mathrm{pc}^{-2}, 1.05 \times\right.\right.$ $\left.\left.10^{10} M_{\odot}, 5.55,0.08,0.035,0.04\right\}\right)$ and the other with median test value equal to $58\left(\left\{\Sigma_{\odot}, M_{\text {bulge }}, c, m_{\mathrm{b}}, j_{\mathrm{d}}, \lambda\right\}=\left\{56.0 M_{\odot} \mathrm{pc}^{-2}\right.\right.$, $\left.\left.9.25 \times 10^{9} M_{\odot}, 6.56,0.09,0.045,0.04\right\}\right)$. As we can see, the difference in the rotation curve between the two models is really negligible, and each set of model with passes the selection procedure has the same statistical weight.

\section{Conclusions}

Modelling the Milky Way is one of the classical tasks of astronomy. In this paper, we have applied the adiabatic compression method in the framework of CDM structure formation to build models of the Galaxy in agreement with observational data and, at the same time, well motivated by numerical simulations of galaxy formation. After exploring the parameter space in great detail, we have finally selected a set of models which has allowed us to place some interesting constraints on: (i) the galactic parameters, namely the Sun distance to the Galactic centre $R_{0}$, the total mass of the bulge $M_{\text {bulge }}$, the disc local surface density $\Sigma_{\odot}$, and the disc scale-length $R_{\mathrm{d}}$; (ii) the parameters entering the adiabatic compression, i.e. the spin parameter $\lambda$, the fraction of the mass in baryons $m_{\mathrm{b}}$, and the thin disc contribution $j_{\mathrm{d}}$ to total angular momentum; and (iii) the parameters describing the initial halo profile (i.e. $c$ and $M_{\text {vir }}$ ).

It would be interesting to compare our results with previous ones in the literature, but a direct comparison is not possible because of the different approaches followed in the analyses. Klypin et al. (2002) have used the adiabatic compression theory to build Milky Way models that agree with observational data starting from an NFW halo. Even if the final aim is the same as ours, the approach is radically different since they give a value for concentration of the initial NFW halo and then find the values of the disc and bulge parameters by trial and errors so that the model is in agreement with the data. In our approach, the visible components are fixed from the beginning and halo parameters are determined later. Klypin et al. (2002) finally examine only four models. Their favoured one is radically different from ours since it has longer disc scale-length (3.5 vs. $2.55 \mathrm{kpc}$ ) and higher concentration (12 vs. 6.48). However, their Model A2 does seem to agree with our results, as it has $c=5$ and $M_{\mathrm{vir}}=7.1 \times 10^{11} M_{\odot}$, and the authors find that this model is also able to fit the data. We stress, however, that most disagreements between our results and those of Klypin et al. (2002) may derive from a different way of selecting the models compatible with the observational constraints.

A somewhat surprising result of our analysis is the fairly small values of concentration parameter $c$ that turns out to be smaller than $11.88(9.75)$ at $95 \%$ for Sample A (Sample B). Using the relation between $M_{\mathrm{vir}}$ and $c$ in Bullock et al. (2001) for values of $M_{\mathrm{vir}}$ in the $95 \%$ range reported in Table 1, one should expect $c$ in the range 14.78-16.04. Considering a $\sim 25 \%$ scatter (Colin et al. 2004), our median $c$ is more than $2 \sigma$ smaller than the lower limit of the predicted range. Moreover, Fig. 6 shows that none of the models have such high values of $c$ that we can conclude that the quoted relation $c-M_{\mathrm{vir}}$ found in numerical simulations is not verified by our observationally selected Milky Way mass models. We have verified that this result is independent on our choice of cosmological parameters by repeating analysis for an Einstein-de Sitter model of universe $\left(\Omega_{\mathrm{M}}=1, \Omega_{\Lambda}=0\right)$. 
Although surprising, this result is not fully unexpected. Fitting adiabatically compressed NFW models to the rotation curve of a sample of 400 spiral galaxies, Jimenez et al. (2003) have determined the values of $c$ and $M_{\mathrm{vir}}$ for these haloes. Looking at their Fig. 1, one sees that there are a lot of galaxies with values of $M_{\mathrm{vir}}$ in the range determined by us for the Milky Way. For these galaxies, the concentration parameter turns out to be on the same order as those obtained here, so that the relation $c-M_{\mathrm{vir}}$ is not satisfied for these galaxies. We are thus confident that the disagreement we have found for the selected Milky Way models is not a result of the our procedure, but a possible shortcoming either of the numerical simulations or of the $\Lambda$ CDM paradigm itself.

The procedure presented may be extended to other spiral galaxies. A possible target is the Andromeda galaxy, whose visible components can be modelled in detail thanks to the available photometric and cinematic data. It is also interesting to apply our procedure to low surface brightness (LSB) galaxies. Detailed modelling of the visible components in these galaxies is very difficult because of the paucity of the data, but these systems are probably dark matter dominated, so that uncertainties in the baryonic components should not systematically affect the main results. Moreover, combining the constraints from many galaxies will allow reduction of the systematics connected with disc modelling. To this aim, a sample of LSB with high resolution rotation curve should be ideal since it allows the disc contribution to be decoupled from the halo one (de Blok \& Bosma 2002).

This analysis may be further refined in order to investigate whether the constraints on the parameters are affected by some simplifying hypotheses. We have implicitly assumed that there is no exchange of angular momentum between the dark matter particles and the baryons infalling in the disc and bulge. An approximate analytical approach to this problem has been developed (Klypin et al. 2002) so it would be interesting to repeat our analysis by including this effect. However, the details of how the baryons are transferred from the halo to the disc depend on the physics of star formation and supernovae explosion, and some kind of energy feedback is needed to eliminate the so-called cooling catastrophe (Balogh et al. 2001). In order to investigate these effects, numerical simulations are needed, but the physics of the process may also be described with semianalytical modelling (Cole et al. 2000; van den Bosch 2002). These methods are best suited to be included in our procedure, thus making it possible to put stronger constraints both on the adiabatic compression parameters $\left(\lambda, m_{\mathrm{b}}, j_{\mathrm{d}}\right)$ and on the initial NFW halo ones $\left(c, M_{\text {vir }}\right)$.

We would like to conclude with a general consideration. The aim of this paper has been to build a Milky Way mass model in agreement with the observational data and motivated by some physical background. The simple adiabatic compression formalism has allowed us to take into account analytically the effect of baryonic infall in a way that is consistent with more complex numerical simulations. We finally found that such a model indeed exists although the median concentration parameter is in disagreement with the one predicted on the basis of numerical simulations. In our opinion, having found such models could be considered as an indirect evidence suggesting that the hierarchical CDM scenario of galaxy formation is essentially correct since its predictions agree with data on the most studied galaxy, the Milky Way. We are thus confident that solutions to problems with this cosmological model do not require modifications of dark matter properties, but have to be sought in astrophysical phenomena.

Acknowledgements. We acknowlege an anonymous referee for having pointed out a major error in our procedure and for his comments that have helped us to improve the paper significantly. We warmly thank Walter Dehnen for having furnished us his code for computing the gravitational potential of the thin and thick discs. It is a pleasure to also thank Shude Mao and Yogesh Joshi for their useful comments.

\section{References}

Alam, S. M. K., Bullock, J. S., \& Weinberg, D. H. 2002, ApJ, 572, 34 Alcock, Ch., Allsman, R. A., Alves, D. R., et al. 2000a, ApJ, 541, 734 Alcock, Ch., Allsman, R. A., Alves, D. R., et al. 2000b, ApJ, 542, 281 Arendt, R. G., Berriman, G. B., Boggess, N., et al. 1994, ApJ, 425, L85

Avelino, P. P., Martins, C. J. A. P., \& Pinto, P. 2002, ApJ, 575, 989

Balogh, M. L., Pearce, F. R., Bower, R. G., \& Kay, S. T. 2001, MNRAS, 326, 1228

Barnes, J., \& Efstathiou, G. 1987, ApJ, 519, 375

Binney, J., \& Evans, N. W. 2001, MNRAS, 327, L27

Binney, J., Gerhard, O. E., \& Spergel, D. N. 1997, MNRAS, 288, 365

Binney, J., \& Tremaine, S. 1987, Galactic dynamics (Princeton University Press)

Blumenthal, G. R., Faber, S. M., Flores, R., \& Primack, J. R. 1986, ApJ, 301, 27

Brand, J., \& Blitz, L. 1993, A\&A, 275, 67

Bullock, J. S., Kolatt, T. S., Sigad, Y., et al. 2001, MNRAS, 321, 559

Bryan, G. L., \& Norman, M. L. 1998, ApJ, 495, 80

Caldwell, J. A. R., \& Ostriker, J. P. 1981, ApJ, 251, 61

Chen, G., \& Ratra, B. 2003, PASP, 115, 1143

Cole, S., Lacey, C. G., Baugh, C. M., \& Frenk, C. S. 2000, MNRAS, 319,168

Colin, P., Klypin, A., Valenzuela, O., \& Gottlöber, S. 2004, ApJ, 612, 50

de Bernardis, P., Ade, P. A. R., Bock, J. J., et al. 2000, Nature, 404, 955

Dame, T. M., Ungerechts, H., Cohen, R. S., et al. 1987, ApJ, 322, 706

de Block, W. J. G., \& Bosma, A. 2002, A\&A, 385, 816

Dehnen, W., \& Binney, J. 1998, MNRAS, 294, 429

Dwek, E., Arendt, R. G., Hauser, M. G., et al. 1995, ApJ, 445, 716

Fall, S. M., \& Efstathiou, G. 1980, MNRAS, 193, 189

Flores, R., Primack, J. R., Blumenthal, G. R., \& Faber, S. M. 1993 , ApJ, 412, 443

Freeman, K. C. 1970, ApJ, 160, 811

Freudenreich, H. T. 1998, ApJ, 492, 495

Gerhard, O. 2002, Mass distribution in our Galaxy, in Matter in the Universe, ed. Ph. Jetzer et al.

Ghigna, S., Moore, B., Governato, F., et al. 2000, ApJ, 544, 616

Gnedin, O. Y., Kravtsov, A. V., Klypin A. A., \& Nagai, D. 2004, ApJ, 616,16

Gott III, J. R., Vogeley, M. S., Podariu, S., \& Ratra, B. 2001, ApJ, 549,1

Jaffe, A. H., Ade, P. A. R., Balbi, A., et al. 2001, Phys. Rev. Lett., 86, 3475

Jesseit, R., Naab, T., \& Burkert, A. 2002, ApJ, 571, L89

Jimenez, R., Verde, L., \& Oh, S. P. 2003, MNRAS, 339, 243

Kent, S. M., Dame, T. M., \& Fazio, G. 1991, ApJ, 378, 131 
Kerr, F. J., \& Lynden-Bell, D. 1986, MNRAS, 221, 1023

Klypin, A., Kravtsov, A. V., Bullock, J. S., \& Primack, J. R. 2001, ApJ, 554, 903

Klypin, A., Kravtsov, A. V., Valenzuela, O., \& Prada, F. 1999, ApJ, 522,82

Klypin, A., Zhao, H. S., \& Somerville, R. S. 2002, ApJ, 573, 597

Kochanek, C. S. 1996, ApJ, 457, 228

Kochanek, C. S. 2002 [arXiv: astro-ph/0108162]

Kuijken, K., \& Dubinski, J. 1994, MNRAS, 269, 13

Kuijken, K., \& Gilmore, G. 1989, MNRAS, 239, 605

Kuijken, K., \& Gilmore, G. 1991, ApJ, 367, L9

Lasserre, T., Afonso, C., Albert, J. N., et al. 2000, A\&A, 355, L39

Lemson, G., \& Kauffmann, G. 1999, MNRAS, 302, 111

Lin, D. N. C., Jones, B. F., \& Klemola, A. R. 1995, ApJ, 439, 652

McGaugh, S., \& de Block, W. J. B. 1998, ApJ, 499, 41

Mo, H. J., Mao, S., \& White, S. D. M. 1998, MNRAS, 295, 319

Moore, B., Governato, F., Quinn, T., \& Stadel, J. 1998, ApJ, 499, L5

Moore, B., Ghigna, S., Governato, F., et al. 1999, ApJ, 524, L19

Navarro, J. F., Frenk, C. S., \& White, S. D. M. 1997, ApJ, 490, 493

Olling, R. P., \& Merrifield, M. R. 2000, MNRAS, 311, 361

Olling, R. P., \& Merrifield, M. R. 2001, MNRAS, 326, 164

Ortiz, R., \& Lépine, J. R. D. 1993, A\&A, 279, 90

Peacock, J. A., Cole, S., Norberg, P., et al. 2001, Nature, 410, 169
Percival, W. J., Baugh, C. M., Bland-Hawthorn, J., et al. 2001, MNRAS, 327, 1297

Pont, F., Queloz, D., Bratschi, P., \& Mayor, M. 1997, A\&A, 318, 416

Popowski, P., Alcock, C., Allsman, R. A., et al. 2000, in Microlensing 2000: A New Era of Microlensing Astrophysics, ed. J. W. Menzies, \& P. D. Sackett, San Francisco, USA, ASP Conf. Proc., 239 [arXiv: astro-ph/0005466]

Reid, M. J. 1993, ARA\&A, 31, 345

Robin, A. C., Crezé, M., \& Mohan, V. 1992, A\&A, 265, 32

Sereno, M. 2003, A\&A, 412, 341

Schmidt, M. 1956, Bull. Astr. Inst. Neth., 13, 15

Sommerville, R. 2002, ApJ, 572, L23

Turner, M. S. 2002, ApJ, 576, L101

van den Bosch, F. C. 2002, MNRAS, 332, 456

Verde, L., Heavens, A. F., Percival, W. J., et al. 2002, MNRAS, 335, 432

Vitvitska, M., Klypin, A., Kravtsov, A. V., et al. 2002, ApJ, 581, 799

Weiland, J. L., Arendt, R. G., Berriman, G. B., et al. 1994, ApJ, 425, L81

Wilkinson, M. I., \& Evans, N. W. 1999, MNRAS, 310, 645

Zaritsky, D. 1999, in the Galactic halo, ed. B. K. Gibbon, T. S. Axelrod, \& M. E. Putnam, San Francisco 